\title{
A way for low ranking matrices and its stochastic computations using Monte Carlo method
}

\author{
BEHROUZ FATHI-VAJARGAH AND ATEYEH JAHANBIN
}

\begin{abstract}
We present approximation theory of matrices based on its low ranking and stochastic computation. Finally, based on our introduced algorithm, we show the performance of these approximations to evaluate the desired results.
\end{abstract}

Mathematics Subject Classification 2000: 65F30

Additional Key Words and Phrases: Low rank, Randomized algorithm, Monte Carlo method, Singular value decomposition, Matrix multiplication.

\section{INTRODUCTION}

In computations, the data may consist of an $\mathrm{m} \times \mathrm{n}$ matrix $\mathrm{A}$ Then, it is often of interest to evaluate a low-rank approximation to A, i.e., an approximation D to the matrix $A$ of rank not bigger than a specified rank $\mathrm{K}$, where $\mathrm{K}$ is smaller than $\mathrm{m}$ and $\mathrm{n}$ Methods such as the singular value decomposition (SVD) can be employed to find an approximation to A which is the best in a well-defined sense [Feigenbaum et al. 1999].

Suppose A and B which are $\mathrm{m} \times \mathrm{n}$ and $\mathrm{n} \times \mathrm{p}$, respectively are the two input matrices. We perform $c$ independent trials, where in each trial we randomly sample an element of $\{1,2, \ldots \mathrm{n}\}$ with an appropriate probability distribution $P$ on $\{1,2, \ldots \mathrm{n}\}$. We form an $\mathrm{m} \times \mathrm{c}$ matrix $\mathrm{C}$ consisting of the sampled columns of A, each scaled appropriately, and we form a $\mathrm{c} \times \mathrm{n}$ matrix $\mathrm{R}$ using the corresponding rows of $\mathrm{B}$, again scaled appropriately. The choice of $\mathrm{P}$ and the column and row scaling are crucial features of the algorithm. When these are chosen, we show that $\mathrm{CR}$ is a good approximation to AB. More precisely, we show that

$$
\|A B-C R\|_{F}=O\left(\|A\|_{F}\|B\|_{F} / \sqrt{c}\right)
$$

Where $\|.\|_{\mathrm{F}}$ denotes the Frobenius norm, i.e., $\|A\|_{F}^{2}=\sum_{i j} A_{i j}^{2}$. This algorithm can be implemented without storing the matrices $A$ and $B$ in $R A M$, provided it can make two passes over the matrices stored in external memory and use $\mathrm{O}(\mathrm{c}(\mathrm{m}+\mathrm{n}+\mathrm{p}))$ additional RAM to construct $C$ and $R$ [Agrawal et al. 1998]. 
We are interested in developing and analyzing fast Monte Carlo algorithms for performing useful computations on large matrices. In this paper we consider the singular value decomposition (SVD); based on two related papers [Drines et al. 2006, pp. 158-183 and pp. 132-157] we consider matrix multiplication and a new method for computing a compressed approximate decomposition of a large matrix. In this paper, we present a computational model for computing on massive data sets (the pass-efficient model) in which our algorithms may naturally be formulated; we also present algorithm for the approximation of the product of two matrices. Also, we present two algorithms for the computation of low-rank approximations to a matrix [Drines et al. 2006, pp. 158-183].

Recent interest in computing with massive data sets has led to the development of computational models in which the usual notions of time efficiency and space efficiency have been modified [Munro and Paterrson. 1978, Henzinger et al. 1998, Achliptas and Mcsherry, Feigenbaum et al. 1999, Drines and Kannan. 2003, Barbara et al. 1997]. In the applications that motivate these data streaming models[Henzinger et al. 1998, Barbara et al. 1997], e.g. the observational sciences and the monitoring and operation of large networked systems, the data sets are much too large to fit into main memory.

\section{APPROXIMATING MATRIX MULTIPLICATION}

Recall that for $\mathrm{A} \in \mathrm{R}^{\mathrm{m} \times \mathrm{n}}$ and $\mathrm{B} \in \mathrm{R}^{\mathrm{n} \times \mathrm{p}}$, a probability distribution $\left\{\mathrm{p}_{1}\right\}_{\mathrm{i}=1}^{\mathrm{n}}$ and a number $\mathrm{c} \leq \mathrm{n}$, it return as out put two matrices, $\mathrm{C}$ and $\mathrm{R}$, such that $\mathrm{CR} \approx A B ; C \in \mathrm{R}^{\mathrm{m} \times \mathrm{c}}$ is a matrix whose columns are $c$ randomly chosen columns of $A$ and $R \in R^{c \times p}$ is a matrix whose rows are the c corresponding rows of B. An important aspect of this algorithm is the probability distribution $\left\{\mathrm{p}_{1}\right\}_{\mathrm{i}=1}^{\mathrm{n}}$ used to choose column-row pairs. Although one could always use a uniform distribution, superior results are obtained if the probability are chosen judiciously. In particular, a set of sampling probabilities $\left\{\mathrm{p}_{1}\right\}_{\mathrm{i}=1}^{\mathrm{n}}$ are the optimal probabilities (with respect to approximating the product $\mathrm{AB}$ ) if they are of form (); for an explanation and discussion, see [Drines et al. 2006, pp. 158-183]. In [Drines et al. 2006, pp. 158-183] is proved the following.

THEOREM 1. Suppose $A \in \mathrm{R}^{\mathrm{m} \times \mathrm{n}}, \mathrm{B} \in \mathrm{R}^{\mathrm{n} \times \mathrm{p}}, \mathrm{c} \in \mathbb{Z}^{+}$such that $1 \leq \mathrm{c} \leq \mathrm{n}$, and $\left\{\mathrm{p}_{1}\right\}_{\mathrm{i}=1}^{\mathrm{n}}$ are such that $\sum_{i=1}^{n} P_{i}=1$ Construct $\mathrm{C}$ and $\mathrm{R}$ with the Matrix Multiplication algorithm of [Drines et al. 2006, pp. 158-183] and let CR be an approximation to AB. If the probability $\left\{\mathrm{p}_{1}\right\}_{\mathrm{i}=1}^{\mathrm{n}}$ are such that 


$$
\mathrm{P}_{\mathrm{k}} \geq \frac{\beta\left|\mathrm{A}^{(\mathrm{k})}\right|\left|\mathrm{B}_{(\mathrm{k})}\right|}{\sum_{\mathrm{k}^{\prime}=1}^{\mathrm{n}}\left|\mathrm{A}^{\left(\mathrm{k}^{\prime}\right)}\right|\left|\mathrm{B}_{\left(\mathrm{k}^{\prime}\right)}\right|}
$$

Then

$$
E\left[\|A B-C R\|_{F}\right] \leq \frac{1}{\sqrt{c}}\|A\|_{F}\|B\|_{F}
$$

If in addition, we let $\sigma \in(0,1)$ and $\eta=1+\sqrt{8 \log (1 / \sigma)}$, Then with probability at least $1-\sigma$,

$$
\|A B-C R\|_{F} \leq \frac{\sigma}{\sqrt{c}}\|A\|_{F}\|B\|_{F}
$$

Furthermore, if the probabilities $\left\{\mathrm{p}_{1}\right\}_{\mathrm{i}=1}^{\mathrm{n}}$ are such that

$$
P_{k}=\frac{\left|B_{(k)}\right|^{2}}{\|B\|_{F}^{2}}
$$

Then

$$
E\left[\|A B-C R\|_{F}\right] \leq \frac{1}{\sqrt{c}}\|A\|_{F}\|B\|_{F}
$$

\subsection{Approximating Matrix Multiplication Algorithms (A.M.M)}

\section{Input:}

$A \in \mathbb{R}^{m \times n}, B \in \mathbb{R}^{n \times p}, c \in \mathbb{Z}^{+}$such that $1 \leq c \leq$

$n$, and $\left\{P_{i}\right\}_{i=1}^{n}$ such that

$P_{i} \geq 0$ and $\sum_{i=1}^{n} P_{i}=1$

Output: $C \in \mathbb{R}^{m \times c}$ and $\mathrm{R} \in \mathbb{R}^{c \times p}$.

1. For $\mathrm{t}=1$ to $\mathrm{c}$,

(a) Pick $i_{t} \in\{1, \ldots, \mathrm{n}\}$ with $\operatorname{Pr}\left[i_{t}=\mathrm{k}\right]=P_{k}, \mathrm{k}=1, \ldots, \mathrm{n}$, independently

and with replacement.

(b) Set $C^{(t)}=A^{\left(i_{t}\right)} / \sqrt{c P_{i_{t}}}$ and $R_{(t)}=B_{\left(i_{t}\right)} / \sqrt{c P_{i_{t}}}$

2. Return $C, R$

When this algorithm is given as input two matrices $A$ and $B$, a probability distribution $\left\{P_{i}\right\}_{i=1}^{n}$, and a number c of column-row pairs to choose, it returns as output matrices $C$ and $R$ such that the product $C R$ is an approximation to $A B$. Observe that since

$$
C R=\sum_{t=1}^{c} C^{(t)} R_{(t)}=\sum_{t=1}^{c} \frac{1}{c P_{(i t)}} A^{\left(i_{t}\right)} B_{\left(i_{t}\right)}
$$




\subsection{Fast Monte-Carlo method for approximating matrix products}

The sequence of matrices $C^{(m)} R_{(m)}, \mathrm{m}=\{1,2, \ldots\}$ where they are independent and identically distribution as $C R$ we call the $C^{(1)} R_{(1)}, C^{(2)} R_{(2)}, \cdots$ perform a sequence of realized simulated matrices of $C R$.

For $\mathrm{m}=\{1,2, \ldots, \mathrm{L}\}, C^{(m)} R_{(m)}$ independent realized stochastic matrices with property $E\left(C^{(m)} R_{(m)}\right)=A B$ i.e. $C^{(m)} R_{(m)}$ with the same distribution as matrix $C R$ where $E\left(C^{(m)} R_{(m)}\right)=A B$, then $G=\frac{1}{L} \sum_{m=1}^{L} C^{(m)} R_{(m)}$ is called the Monte Carlo simulated matrix of $C R$, Table I shows evaluate an approximation of $A B$ by Monte Carlo method where $C$ and $R$ have the same structure as explained in $A . M . M$ method. For the example let $A \in \mathbb{R}^{m \times n}, B \in \mathbb{R}^{n \times p}, c=2$.

Table 1. Relative error of example results

\begin{tabular}{|l|c|c|c|c|c|}
\hline$m, n, p$ & $\mathbf{1 0}$ & $\mathbf{5 0}$ & $\mathbf{1 0 0}$ & $\mathbf{1 5 0}$ & $\mathbf{2 0 0}$ \\
\hline$m, n=500, p=300$ & 0.0402 & 0.0074 & 0.0033 & 0.0027 & 0.0021 \\
\hline$m, n=1000, p=900$ & 0.0394 & 0.0074 & 0.0038 & 0.0027 & 0.0019 \\
\hline$m, n=1500, p=1000$ & 0.0382 & 0.0076 & 0.0037 & 0.0024 & 0.0020 \\
\hline$m, n=2000, p=1000$ & 0.0373 & 0.0075 & 0.0038 & 0.0025 & 0.0020 \\
\hline
\end{tabular}

It is well known that the relative error is $\frac{\|A B-C R\|_{F}}{\|A\|_{F}\|B\|_{F}}$.

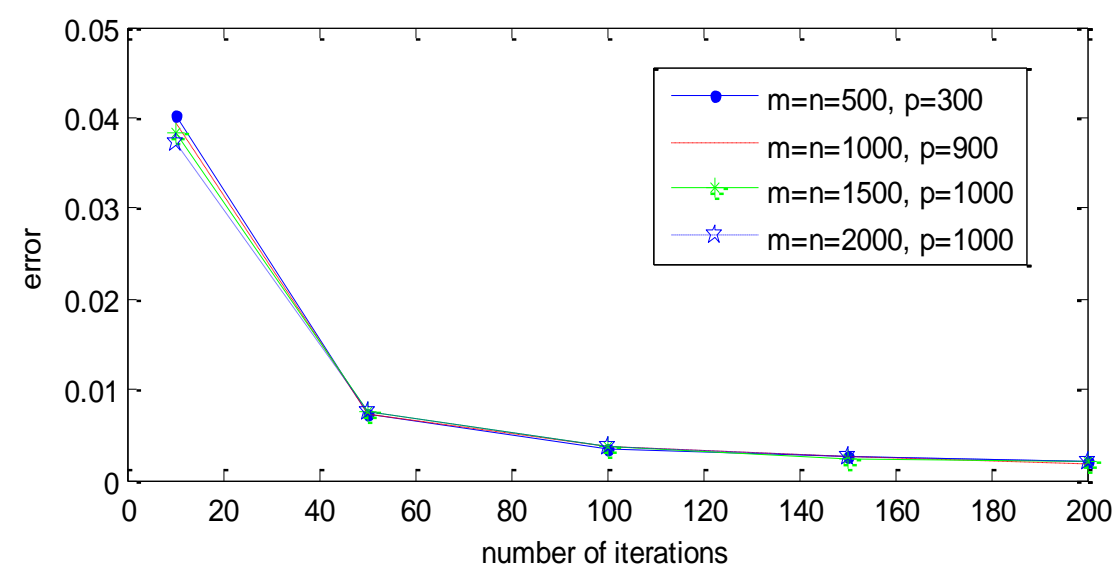

Fig. 1. Error of results based on number of iterations based on A. M. M Algo 


\subsection{Implementation of The sampling and running time.}

To implement the A.M.M algorithm, it must be decided which elements of the input to sample and those elements must then be sampled. In the case of uniform sampling one can decide before the input is seen which column-row pairs to sample. Then, a single pass over the matrices is sufficient to sample the columns and rows of interest and to construct $C$ and $R$; this requires $O(c(m+p))$ additional time and space. We will see below that it is useful to sample according to a non uniform probability distribution that depends on column and row lengths, e.g.

In order to decide which column-row pairs to sample in such a case, one pass through the matrices and $O(n)$ additional time and space is sufficient; in the additional space running totals of $\left|A^{(k)}\right|^{2}$ and $\left|B_{(k)}\right|^{2}$ are kept, so that after the first pass $\left|A^{(k)}\right|,\left|B_{(k)}\right|$, $k=1, \ldots, n$, and thus the probabilities, can be calculated in $O(n)$ additional time.

Then in a second pass the columns and rows of interest can be sampled and $C$ and $R$ can be constructed and stored; this requires $O(c(m+p))$ additional space and time. Thus, in addition to either one or two passes over the data, for both uniform and non uniform sampling, $O(c(m+n+p))$ additional space and time is sufficient to sample from the matrices $A$ and $B$ of the input and to construct the matrices $C$ and $R$.

In Table II, we compare the computational time needed for approximating $A B$ by $C R$ multiplication, using $c=2, L=200$ and the corresponding time needed for obtaining $A B$ multiplication where we show them by time 1 and time 2 , respectively.

Table 2. Computational time for $\mathrm{CR}$ and $\mathrm{AB}$

\begin{tabular}{|c|c|c|c|}
\hline $\mathrm{m}, \mathrm{n}, \mathrm{p}$ & time 1 & time 2 & Relative Error \\
\hline$m, n=500, p=300$ & 1.6869 & 3.909653 & 0.0021 \\
\hline$m, n=1000, p=900$ & 4.812916 & 50.051374 & 0.0019 \\
\hline$m, n=1500, p=1000$ & 7.815693 & 125.800965 & 0.0020 \\
\hline$m, n=2000, p=1000$ & 10.503226 & 249.492549 & 0.0020 \\
\hline
\end{tabular}




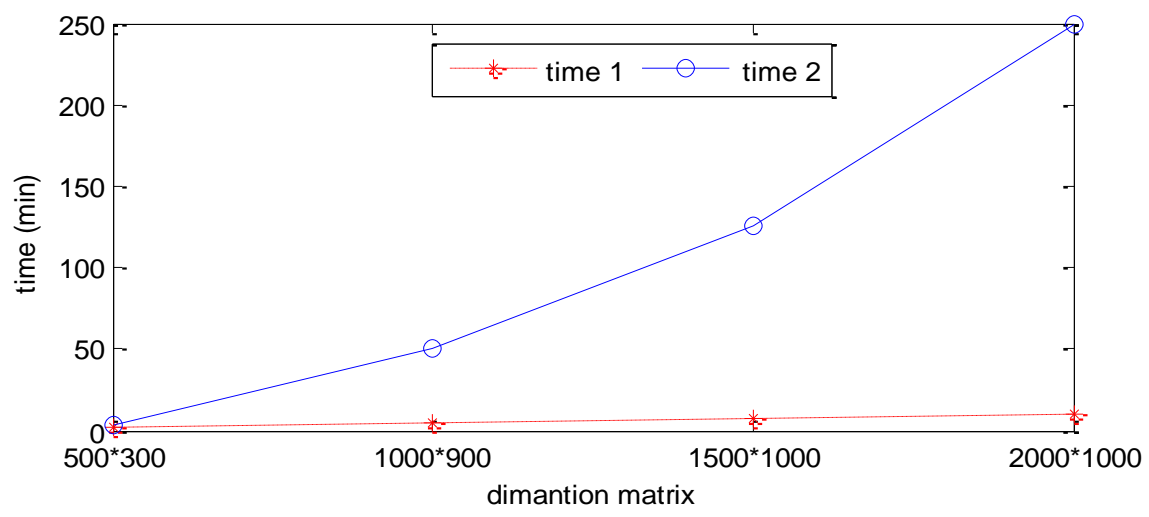

Fig. 2. Comparison of computational time for obtaining $C R$ and $A B$

We note that the computational time for obtaining $C R$ based on algorithm A.M.M is significantly less than the corresponding time for evaluating $A B$.

\section{THE SINGULAR VALUE DECOMPOSITION (SVD)}

If $A \in \mathbb{R}^{m \times n}$, then there exist orthogonal matrices $U \in \mathbb{R}^{m \times m}$ and $V \in \mathbb{R}^{n \times n}$ and $U^{T} A V=$ $\Sigma$ and $\Sigma$ is a diagonal matrix $m \times n$ such that

$$
\begin{gathered}
U^{T} A V=\Sigma=\operatorname{diag}\left(\sigma_{1}, \sigma_{2}, \cdots, \sigma_{\rho}\right), \rho=\min \{m, n\} \\
\sigma_{1} \geq \sigma_{2} \geq \cdots \geq \sigma_{\rho} \geq 0
\end{gathered}
$$

Then the $S V D$ decomposition of $A$ is given as $A=U \Sigma V^{T}$. The vectors $u^{i}, v^{i}$ are called the left and right singular vectors of $A$ respectively, which correspond to the singular value $\sigma_{i}$. The left and the right singular vectors can be computed from the right and the left singular vectors by the formulas:

$$
A v^{i}=\sigma_{i} u^{i} \quad A^{T} u^{i}=\sigma_{i} v^{i}
$$

Equivalently, $\sigma_{1}^{2} \geq \sigma_{2}^{2} \geq \cdots \geq \sigma_{r}^{2}>0$ are all the positive eigenvalues of the nonnegative definite symmetric matrices $A A^{T}, A^{T} A$, with the corresponding orthonormal eigenvectors $u_{1}, u_{2}, \cdots, u_{r} \in \mathbb{R}^{m}$ and $v_{1}, v_{2}, \cdots, v_{r} \in \mathbb{R}^{n}$.

we define $\mathrm{r}$ by $\sigma_{1} \geq \sigma_{2} \geq \cdots \geq \sigma_{r}>\sigma_{r+1}=\cdots=\sigma_{\rho}=0$, then $\operatorname{rank}(\mathrm{A})=\mathrm{r}$, and $\operatorname{rang}(A)=\operatorname{span}\left(u^{1}, \cdots, u^{r}\right) \quad \operatorname{null}(A)=\operatorname{span}\left(v^{r+1}, \cdots, v^{\rho}\right) . \quad$ If $\quad$ we let $U_{r} \in \mathbb{R}^{m \times r}$ denote the matrix consisting of the first $\mathrm{r}$ columns of $\mathrm{U}, V_{r} \in \mathbb{R}^{r \times n}$ denote the matrix consisting of the first $r$ columns of $V$, and $\Sigma_{r} \in \mathbb{R}^{r \times r}$ denote the principal $r \times r$ sub matrix of $\Sigma$, then

$$
A=U_{r} \Sigma_{r} V_{r}^{T}=\sum_{t=1}^{r} \sigma_{t} u^{t} v^{t^{T}}
$$


Note that this decomposition property provides a canonical description of a matrix as a sum of $r$ rank-one matrices of decreasing importance. If $k \leq r$ and we define

$$
\begin{gathered}
A_{k}=U_{k} \Sigma_{k} V_{k}^{T}=\sum_{t=1}^{k} \sigma_{t} u^{t} v^{t^{T}}, \quad A_{k}=U_{k} U_{k}^{T} A=\left(\sum_{t=}^{k} u^{t} u^{t^{T}}\right) A, \\
A_{k}=A V_{k} V_{k}^{T}=A\left(\sum_{t=1}^{k} v^{t} v^{t^{T}}\right)
\end{gathered}
$$

$A_{k}$ is the projection of $A$ onto the space spanned by the top k singular vectors of $A$.

Furthermore, the distance between $A$ and any rank- $k$ approximation to $A$ is minimized by, $A_{k}$ i.e.,

$$
\begin{aligned}
& \min _{D \in R^{m \times n}: \operatorname{rank}(D) \leq k}\|A-D\|_{F}^{2}=\left\|A-A_{k}\right\|_{F}^{2}=\sum_{t=k+1}^{r} \sigma_{t}^{2}(A) \\
& \left\|A_{k}\right\|_{F}^{2}=\sum_{t=1}^{k} \sigma_{t}^{2} \quad, \quad\|A\|_{F}^{2}=\sum_{t=1}^{r} \sigma_{t}^{2} .
\end{aligned}
$$

\section{LINEAR TIME SVD APPROXIMATION}

Given a matrix $A \in \mathbb{R}^{m \times n}$ we wish to approximate its top $k$ singular values and the corresponding singular vectors. The strategy behind the Linear Time SVD algorithm is to pick $c$ columns of the matrix $A$, rescale each by an appropriate factor to form a matrix $C \in \mathbb{R}^{\mathrm{m} \times \mathrm{n}}$, and then compute the singular values and corresponding left singular vectors of the matrix $C$, which will be approximations to the singular values and left singular vectors of $A$, in a sense we make precise later. These are calculated by performing an $S V D$ of the matrix $C C^{T}$ to compute the right singular vectors of $C$ and from them calculating the left singular vectors of $C$.

It will be shown that if the probabilities $\left\{P_{i}\right\}_{i=1}^{n}$ are chosen judiciously, then the left singular vectors of $C$ are with high probability approximations to the left singular vectors of $A$.

We will show that in addition to this error the matrix $H_{k} H_{k}^{T} A$ has an error that depends on $\left\|A A^{T}-C C^{T}\right\|_{\mathrm{F}}$ then, using the results of THEOREM 1, we will show that this additional error depends on $\|A\|_{\mathrm{F}}^{2}$.

THEOREM 2. Suppose $A \in \mathbb{R}^{m \times n}$ and let $H_{k}$ be constructed from the L.T.SVD algorithm. Then [Drines et al. 2006, pp. 132-157],

$$
\left\|A-H_{k} H_{k}^{T} A\right\|_{F}^{2} \leq\left\|\mathrm{A}-\mathrm{A}_{\mathrm{k}}\right\|_{\mathrm{F}}^{2}+2 \sqrt{k}\left\|A A^{T}-C C^{T}\right\|_{\mathrm{F}} .
$$

THEOREM 3. Suppose $A \in \mathbb{R}^{m \times n}$; let $H_{k}$ be constructed from the L.T.SVD algorithm by sampling c columns of $A$ with probabilities $\left\{P_{i}\right\}_{i=1}^{n}$ such that $p_{i} \geq$ 
$\beta\left|A^{(i)}\right|^{2} /\|A\|_{F}^{2}$ for some positive $\beta \leq 1$, and let $\eta=1+\sqrt{8 / \beta \log (1 / \delta)}$. let $\varepsilon>0$. If $c \geq 4 k / \beta \varepsilon^{2}$, then [Drines et al. 2006, pp. 132-157],

$$
E\left[\left\|A-H_{k} H_{k}^{T} A\right\|_{F}^{2}\right] \leq\left\|A-A_{k}\right\|_{F}^{2}+\varepsilon\|A\|_{\mathrm{F}}^{2}
$$

and if $c \geq 4 k \eta^{2} / \beta \varepsilon^{2}$, then with probability at least $1-\sigma$,

$$
\left\|A-H_{k} H_{k}^{T} A\right\|_{F}^{2} \leq\left\|\mathrm{A}-\mathrm{A}_{\mathrm{k}}\right\|_{\mathrm{F}}^{2}+\varepsilon\|A\|_{\mathrm{F}}^{2} .
$$

\section{1. $\quad$ Linear Time SVD Algorithm (L.T.SVD)}

\section{Input: $\quad A \in \mathbb{R}^{m \times n}, B \in \mathbb{R}^{n \times p}, c, k \in \mathbb{Z}^{+}$such that $1 \leq k \leq c \leq$} $n$, and $\left\{P_{i}\right\}_{i=1}^{n}$ such that

$P_{i} \geq 0$ and $\sum_{i=1}^{n} P_{i}=1$

Output: $H_{k} \in \mathbb{R}^{m \times n}$ and $\sigma_{t}(C), t=1, \cdots, k$.

1. For $\mathrm{t}=1$ to $\mathrm{c}$,

(a) Pick $i_{t} \in 1, \ldots, \mathrm{n}$ with $\operatorname{Pr}\left[i_{t}=\alpha\right]=P_{\alpha}, \alpha=1, \ldots, \mathrm{n}$.

(b) Set $A^{\left(i_{t}\right)} / \sqrt{c P_{i_{t}}}$.

2. Compute $C^{T} C$ and its SVD; say $C^{T} C=\sum_{t=1}^{c} \sigma_{t}^{2}(C) y^{t} y^{t^{T}}$

3. Compute $h^{t}=C y^{t} / \sigma_{t}(C)$ for $\mathrm{t}=1, \ldots, \mathrm{k}$.

4. Return $H_{k}$, where $H_{k}^{(t)}=h^{t}$, and $\sigma_{t}(C), \mathrm{t}=1, \ldots, \mathrm{k}$.

\subsection{Implementation details and running time}

To measure the approximation error we defined the relative error of the approximation as, $\left\|A-H_{k} H_{k}^{T} A\right\|_{F}^{2} /\|A\|_{F}^{2}$ where $A$ is the original data matrix and $H_{k} H_{k}^{T} A$ is k-rank approximation to $A$ given by L.T.SVD algorithm. the optimal error $\left\|A-A_{k}\right\|_{F}^{2} /\|A\|_{F}^{2}$ where $A_{k}$ is the optimal $k$-rank approximation to matrix $A$. The best approximation is given by singular value decomposition, which is too time consuming for very large $m$ and $n$.

Given the elements to be sampled, the matrix $C$ can then be constructed in one additional pass; this requires additional space and time that is $O(m c)$. Given $C \in \mathbb{R}^{m \times c}$, computing $C^{T} C$ requires $O(m c)$ additional space and $O\left(m c^{2}\right)$ additional time, and computing the $S V D$ of $C^{T} C$ requires $O\left(c^{3}\right)$ additional time. Then computing $H_{k}$ requires k matrix-vector multiplications for a total of $O(m c k)$ additional space and time. Thus, overall $O\left(c m+c^{2}\right)$ additional space and $O\left(c^{2} m+c^{3}\right)$ additional time are required by the L.T.SVD algorithm. 


\section{SIMULATION RESULTS}

To assess the performance of $\mathrm{k}$ rank approximation algorithm we conducted different simulation on synthetic data. We also implemented our algorithm for three different sampling methods:

1) Uniform sampling with out replacement. we removed the sampled columns after sampling. Thus each column can be sampled at most once.

2) Uniform sampling with replacement. We did not remove the sampled columns after sampling. Thus some columns were sampled more than once.

3) Weighted sampling. Thus a row had a bigger weight if it differed more from its neighbor. The columns weight is calculated by $z_{j}=\sum_{j=1}^{n} \sum_{i=1}^{m}|A(i, j)|^{2}$.

To measure the approximation error we defined the relative error of the approximation as $\left\|A-H_{k} H_{k}^{T} A\right\|_{F}^{2} /\|A\|_{F}^{2}$, where $\mathrm{A}$ is the original data matrix and $H_{k} H_{k}^{T} A$ is k-rank approximation to A given by L.T. SVD algorithm and Relative error of optimal $k$ rank approximation is $\left\|A-A_{k}\right\|_{F}^{2} /\|A\|_{F}^{2}$, where $A_{k}$ is the optimal true k-rank approximation to matrix A.

Figure 3 shows the performance of L.T.SVD algorithm by Monte Carlo method for a randomly generated data matrix $A \in R^{200 \times 200}$ with full rank. We were looking for rank $\mathrm{k}=1$ approximation of $\mathrm{A}$. In each iteration we randomly picked $\mathrm{c}=50$ columns of the data matrix with and without replacement. The optimum relative error is $\left\|A-A_{k}\right\|_{F}^{2} /\|A\|_{F}^{2} \approx$ 0.2474 . As we expected the convergence property is faster when the columns are sampled without replacement. We see that 15 iterations give a very good approximation to the optimal solution $A_{1}$, Table III shows experimental results.

Figure 4 shows the performance of L.T.SVD algorithm for a randomly generated data matrix $A \in R^{1500 \times 1500}$ with full rank. We were looking for rank k=1 approximation of A. Using a fixed $k=1$ and increasing $c$ (the number of columns that we select them randomly), we want to evaluate the relative error, the computational time of both L.T.SVD and optimal SVD algorithms and compare them together. We note that for the relative error and speed of optimal are 0.2497 and $203.215822 \mathrm{~min}$, respectively, Table IV shows experimental results.

Figure 5 shows the computational compare time using Monte Carlo (L.T.SVD) and optimal SVD algorithms. 


\section{CONCLUSIONS}

We have proposed a novel approach for fast computing of $\mathrm{k}$ - rank approximation of a given $\mathrm{m} \times \mathrm{n}$ data matrix, using Monte-Carlo method by choosing at random $\mathrm{c}$ columns of A.

The advantage of the algorithm is that we guarantee that every iteration improves the quality of our approximation. Our results confirm the convergence of the relative errors of the approximation to the optimal relative error. To high light the Important feature of this algorithm we applied this method on a big Matrix of randomly generated data. We observed for the reasonable level of relative error the algorithm is much faster than optimal k-rank approximation using deterministic SVD, which may also fail to compute the SVD for big matrices.

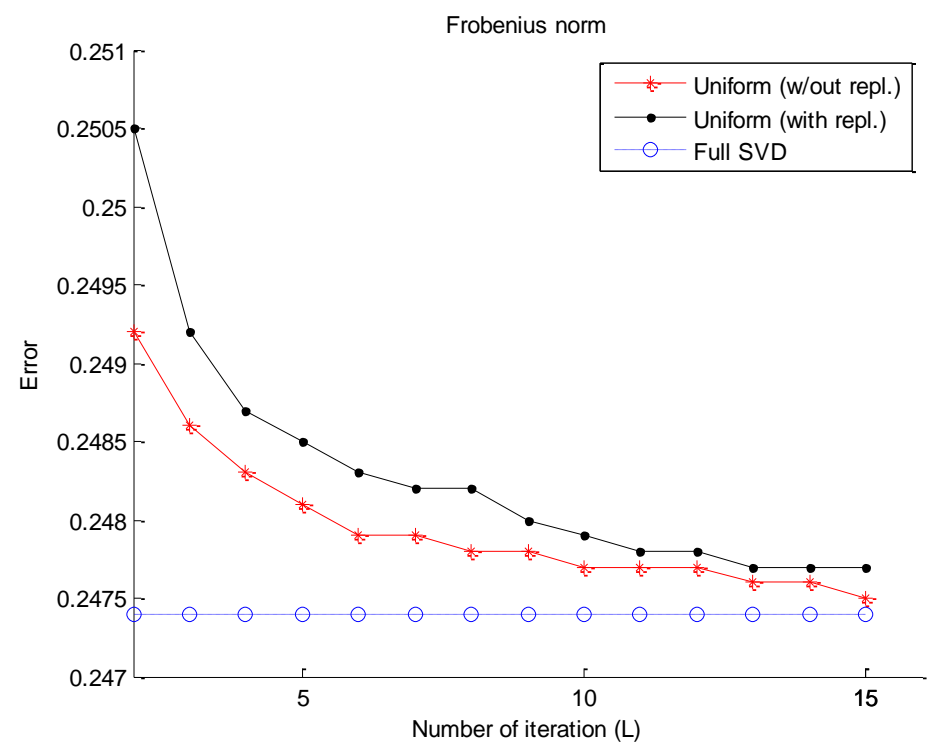

Fig. 3. Convergence property of the Monte-Carlo method for Random data matrix $(200 \times 200), \mathrm{c}=50, \mathrm{k}=1$. 


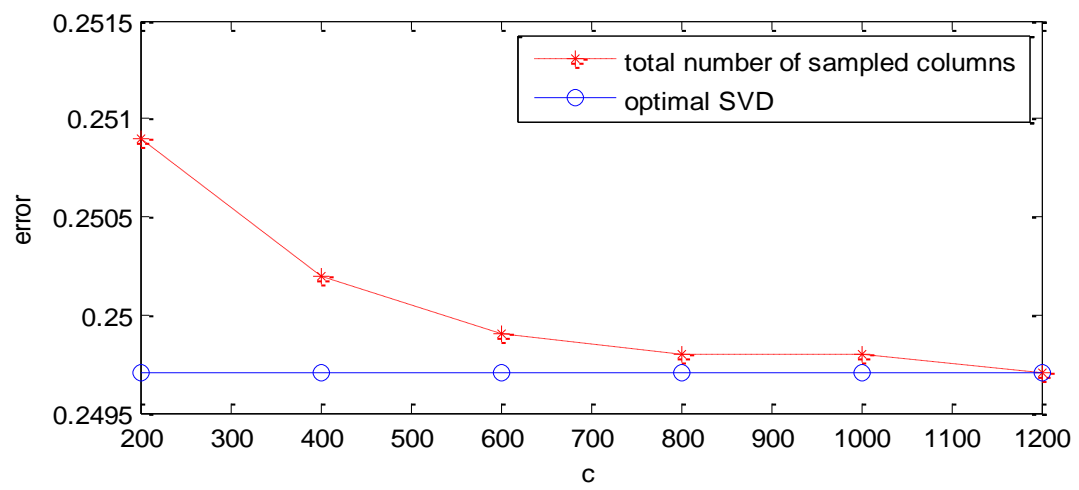

Fig. 4. Error of results for optimal $S V D$ algorithm based on $c$

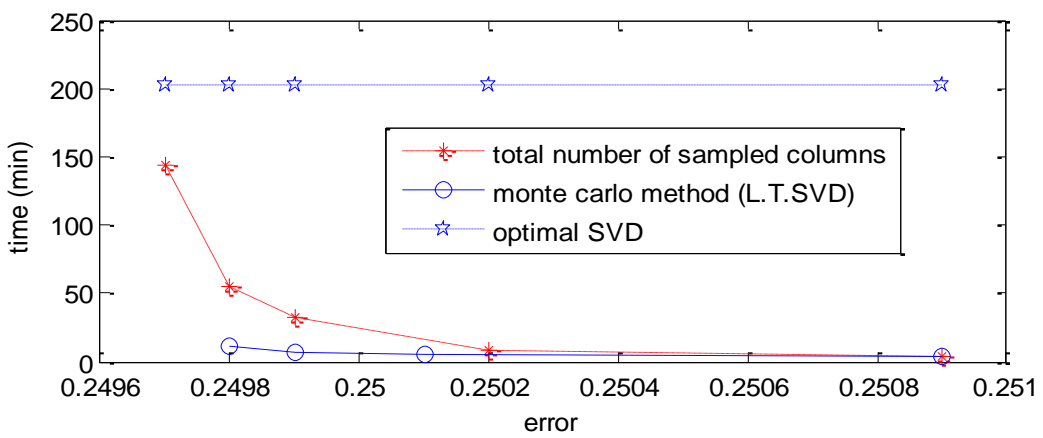

Fig. 6. comparison of need time for approximation using MC and SVD methods.

Table 3. Error of results for Monte Carlo algorithm based on L

\begin{tabular}{|c|c|c|}
\hline$L$ & $\frac{\left\|A-H_{k} H_{k}^{T} A\right\|_{F}^{2}}{\|A\|_{F}^{2}}$ & $\frac{\left\|A-H_{k} H_{k}^{T} A\right\|_{F}^{2}}{\|A\|_{F}^{2}}$ \\
& $($ Uniform $($ w /out repl $))$ & (Uniform(with repl). $)$ \\
\hline 2 & 0.2492 & 0.2505 \\
\hline 4 & 0.2483 & 0.2487 \\
\hline 6 & 0.2479 & 0.2483 \\
\hline 8 & 0.2478 & 0.2482 \\
\hline 10 & 0.2477 & 0.2479 \\
\hline 12 & 0.2477 & 0.2478 \\
\hline 13 & 0.2476 & 0.2477 \\
\hline 14 & 0.2476 & 0.2477 \\
\hline 15 & 0.2475 & 0.2477 \\
\hline
\end{tabular}


Table 4. Relative error and computational time of optimal SVD algorithm

\begin{tabular}{|c|c|c|}
\hline$c$ & Relative Error & Time \\
\hline 200 & 0.2509 & 3.720119 \\
\hline 400 & 0.2502 & 8.071144 \\
\hline 600 & 0.2499 & 31.635689 \\
\hline 800 & 0.2498 & 55.630737 \\
\hline 1000 & 0.2498 & 91.607197 \\
\hline 1200 & 0.2497 & 144.304089 \\
\hline
\end{tabular}

\section{REFERENCES}

ACHLIOPTAS. D AND MCSHERRY. F, Fast computation of low rank matrix approximations, J.ACM, to appear. Agrawal. R, Gerhrke. J, Gunopulos. D, and Raghavan. P, Automaticsubspace clustering of high dimensional data for data mining applications, Proc. ACM SIGMOD Conf. on Management of Data, 1998, 94-105.

Barbara. D, Faloutsos. Hellerstein. C, Ioannidis.Y, Jagadish. H. V., Johnson. T, R. NG, Poosala.V, Ross. K, AND SeVcIK. K. C, The New Jersey data reduction report, Bulletin of the IEEE Computer Society Technical Committee on Data Engineering, 1997.

Drineas. P, KAnNAN. R, And Mahoney. M. W, Fast Monte Carlo algorithms for matrices I:Approximating matrix multiplication, SIAM J. Comput., 36 (2006), pp. 132-157.

DrineAs. P, KANNAN. R, AND MAHONEY. M. W, Fast Monte Carlo algorithms for matricesII: Computing a low-rank approximation to a matrix, SIAM J. Comput., 36 (2006), pp.158-183.

DRINEAS. P AND KANNAN. R, Pass efficient algorithms for approximating large matrices, in Proceedings of the 14th Annual ACM-SIAM Symposium on Discrete Algorithms, 2003,pp. 223-232.

Feigenbaum. J, Kannan. S, Strauss. M, and Viswanathan. M, An approximate L1-difference algorithm for massive data sets, in Proceedings of the 40th Annual IEEE Symposium on the Foundations of Computer Science, 1999, pp. 501-511.

Golub. G. H and Van Loan. C. F, Matrix Computations, Johns Hopkins University Press, London,1989.

Henzinger. M. R, Raghavan. P, And Rajagopalan. S, Computing on Data Streams, Tech. Report 1998011, Digital Systems Research Center, Palo Alto, CA, 1998.

Munro. J. I And PATERson. M. S, Selection and sorting with limited storage, in Proceedings of the 19th Annual IEEE Symposium on Foundations of Computer Science, 1978, pp. 253-258.

Behrouz Fathi-Vajargah, Department of Statistics, The University of Guilan, Iran e-mail: fathi@guilan.ac.ir.

Ateyeh Jahanbin, Department of Statistics, The University of Guilan, Iran. at.jahanbin@yahoo.com 